\title{
The people's camera
}

\author{
Taxpayer-funded scientific research carries with it the responsibility to communicate to the general \\ public its relevance in an accessible, open and inspirational manner. The HiRISE project that steers a \\ camera on Mars admirably achieves this aim.
}

The global recession has caused many governments to reassess their spending priorities. With tightened budgets and mounting competition for funding, it becomes increasingly important to justify the use of the taxpayers' money in supporting specific scientific research projects. Increased democratic engagement with scientific practice is one way to achieve this.

In one such enterprise of engaging the public, the team in charge of the martian High Resolution Imaging Science Experiment (HiRISE) launched HiWish on 20 January 2010, a web-based platform that allows the public to register and vote for sites they wish the camera to visit (www.uahirise.org/suggest). HiRISE is a camera on board the Mars Reconnaissance Orbiter that captures high-resolution images of Mars's surface. Such imagery allows viewing the martian surface in the greatest detail possible, similar to (and in some places better than) Google satellite imagery of Earth. The data are useful for measurements and geological modelling, and are required to support sample return and surface exploration for future Mars landers.

HiWish, dubbed The People's Camera, will continue until at least the end of 2011. But those who believe that science is not safe in the hands of the uninitiated should worry not. Suggestions must be accompanied by a justification and final decisions on data collection are made by a team of specialists. Owing to the high resolution of the imagery, HiRISE will only be able to capture $2 \%$ of the martian surface. Every image must count.

Over 3,000 people are registered with HiWish and, by mid-February 2010, over 700 suggestions had already been received. This level of interest attests the project's accessibility, relevance and ability to ignite passion. Registration is via the internet, with translation available in several languages. Progress can be tracked through social networking sites. Furthermore, the images are completely open access, to be used in any capacity imaginable. At present the team are working to produce stereopair images, combined with Digital Terrain Models, allowing three-dimensional

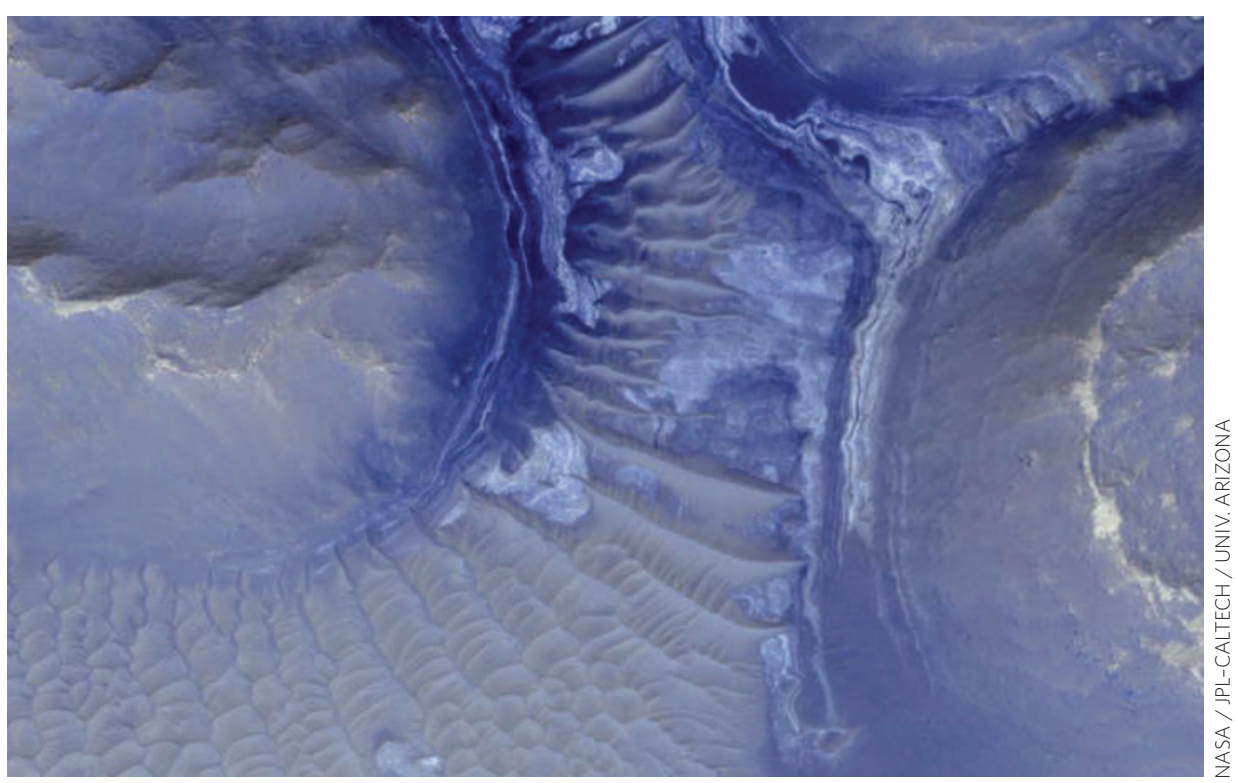

Noctis Labyrinthus on Mars, seen from the High Resolution Imaging Science Experiment (HiRISE) camera on NASA's Mars Reconnaissance Orbiter.

tours through martian valleys, channels, volcanoes and craters.

As one blogger states on the HiRISE website, HiWish is to an amateur planetary enthusiast what being given the chance to score in the Superbowl would be to a football fan. And the project comes well timed to highlight research on Mars towards the end of the life of the martian rovers Spirit and Opportunity (Nature 463, $600 ; 2010)$ that have benefitted from keen public interest.

The costs of building and running the HiRISE mission so far are estimated at around $\$ 70$ million. As the team points out, this amounts to just 22 cents per American citizen. Whether HiRISE will continue collecting data in a follow-up mission depends on the outcome of the full USA budget report, but the inspirational value of the project for a future generation of planetary scientists cannot be quantified in monetary terms.

It is not only the amateurs who benefit from participatory science. The resources of the public's time, enthusiasm and computer power have been used in a variety of scientific endeavours. In the Galaxy Zoo, launched in 2007, volunteer astronomers help with the classification of millions of images of galaxies (www.galaxyzoo.org). The Springwatch Survey, run between 2005 and 2007 by the UK's BBC and Woodland Trust (and now continuing as Nature's Calendar), encourages the UK public to monitor and record key events indicative of the onset of spring, such as the first signs of frogspawn, hawthorn blossom or the arrival of swifts (www.naturescalendar.org.uk). Distributed computing, where many members of the public donate idle time on their computers for a common project, has aided the search for extraterrestrial intelligence (http://setiathome.berkeley.edu/), and helped explore the uncertainty range of climate models (http://climateprediction.net/).

Projects that engage the public with science take research administration to a new level of complexity that needs careful management. But tapping the creativity and talent of individuals, while promoting a broad understanding of scientific thinking is worth the effort. 